\title{
Research on Economic Mathematical Analysis and Construction Model of Prefabricated Building Structure Based on Improved Neural Network Algorithm
}

\author{
Xin Lin (i) \\ School of Urban Construction Engineering, Chongqing Radio \& TV University, Chongqing, China \\ Correspondence should be addressed to Xin Lin; linxin49840566@163.com
}

Received 13 August 2020; Revised 26 November 2020; Accepted 21 January 2021; Published 17 April 2021

Academic Editor: Jia-Bao Liu

Copyright (c) 2021 Xin Lin. This is an open access article distributed under the Creative Commons Attribution License, which permits unrestricted use, distribution, and reproduction in any medium, provided the original work is properly cited.

\begin{abstract}
In this article, a mathematical analysis model of economics of prefabricated building structure based on improved neural network algorithm is proposed in order to solve the low analysis accuracy in traditional methods. Firstly, by means of analyzing the costs of materials, labor, and equipment, the economic characteristics of the cost of fabricated building structures are determined. Secondly, the single neuron is analyzed and the weight coefficient is adjusted in accordance with the multilayer neural network structure, so as to minimize the construction error of the economic analysis model of the assembled building structure. Meanwhile, the weight vector is obtained, error-weighted square sum is calculated through choosing an adaptive filter and obtained, and the weight vector is updated by the least squares algorithm. Thirdly, the neural network algorithm training and learning process is designed and improved, the dependent variable is selected, the number of input points is determined, and then, the training and learning process of the improved neural network algorithm is completed. Finally, a fitness function is set to measure the authenticity of dataset, which is further defined as a combination of different weights to construct an economic mathematical analysis model. The experimental results indicate that the analysis results of this method can reach an accuracy up to $96 \%$, so it has a broader application prospect in low-rise buildings.
\end{abstract}

\section{Introduction}

Nowadays, as social progress, the prefabricated building structures with low capital, simple structure, and good performance are in urgent need [1]. Compared with traditional concrete building structures, the buildings adopting prefabricated structural systems are better in terms of antiearthquake strength, firefighting level, corrosion resistance, and construction waste production [2]. Meanwhile, by virtue of a shorter construction period, the prefabricated structure buildings not only can greatly reduce the construction cost but also is more practical than the concrete building structure [3]. Considering the large number of floors, large area, and high manpower as well as material resources required for superhigh-rise buildings [4], the structure cost accounts for $1 / 3$ of the total price. Therefore, it is of great significance to conduct economic analysis on the fabricated building structure [5]. Although prefabricated building structures are advantageous in low carbon emissions, green environmental protection, and sustainable development, there are only a few researches focusing on the economic mathematical analysis of prefabricated building structures [6]. In response to this problem, the research from the perspective of structural economy is carried out based on the example of actual project [7]. As an artificial intelligence technology, neural network has received many experts' studies in the field of economic analysis, and a good analysis result is achieved [8]. However, there are still some problems such as insensitive to the initial spatial conditions of high dimensions and multiple local minima, so it is necessary to search for global data points by using as few control parameters as possible [9]. To this end, a mathematical analysis model for the economics of fabricated building structures based on improved neural network algorithms is 
constructed, which can provide engineering companies with a scientific basis for grasping future economic development trends [10].

\section{Economic Characteristics of Fabricated Building Structure}

The cost of prefabricated building structure is mainly composed of direct and indirect engineering costs, in which the direct engineering costs include labor costs, material costs, equipment costs, site management fees, and construction costs [11]; indirect engineering costs contain material expenses, utilities, maintenance fees, transportation fees, installation fees, and measures fees [12].

2.1. Material Expenses. By fully taking into account the lifting requirements, prefabricated building structure generally maximizes the amount of components so that the amount of building materials has also increased [13]. In order to save money to the greatest extent during the production process, prefabricated building structure uses a higher turnover template to replace the wooden template. This means the consumption of prefabricated building structure materials and material expenses will be greatly reduced if the standardization level of the prefabricated building structure and the turnover frequency of the formwork are improved [14].

2.2. Labor Costs. The labor costs of prefabricated building structures mainly include labor costs in the production process, transportation costs, and labor costs in installation. Due to the continuous development of industrial production measures and processes, concrete building structures that require longer working hours for workers are gradually reduced [15].

2.3. Equipment Costs. Equipment costs are the major factor impeding the economic progress of fabricated building structures. The diversification of China's current buildings has generated a higher demand for fabricated building structures, which results in an increase in the cost of traditional concrete building structures, the one-time investment in equipment, and the pressure of equipment cost recovery [16].

\section{Improved Neural Network Algorithm}

The structure of multilayer neural network is shown in Figure 1.

The neuron structure of the neural network is shown in Figure 2.

The neural network aims to adjust the weight coefficients to ensure a minimized construction error of the economic mathematical analysis model of fabricated building structure [17]. Each neuron is connected by a linear combination and a nonlinear function. Since the linear combination has the same structure as the adaptive filter, the least squares algorithm is applicable to update the weight vector [18]. The specific process is as follows.

First, an adaptive filter is selected, and the weight vector is obtained as follows:

$$
Q(n)=\left[Q_{0}(n), Q_{1}(n) \Delta Q_{n-1}(n)\right]^{T}
$$

The data vector is input:

$$
f(n)=[f(n), f(n-1) \Delta f(n+1)]^{T} .
$$

The objective function is the error weighted sum of squares, and the specific calculation content is as follows:

$$
Z(n)=\sum_{i=1}^{n} \alpha^{n-1}(d(i)-Q(n) f(i))^{2} .
$$

In formula (3), $\alpha$ represents the forgetting factor and $d(i)$ represents the expected output value.

In this algorithm, the expected value output by using the neuron is affected by the multilayer structure, and it is unable to measure the expected response of the middle layer neuron, so the error backpropagation shall be adopted to calculate the expected response value [19].

The specific steps of the improved neural network algorithm are as follows:

Step 1: the weights are initialized, and the learning step length is determined to ensure that the forgetting factor is within the error range.

Step 2: the output value of the linear combination of each layer of the network is calculated according to the forward propagation of input mode.

Step 3: the weights are updated through the least square algorithm, and the output error is checked to complete the improvement of neural network algorithm.

\section{Application of Improved Neural Network Algorithm in Mathematical Analysis Model}

4.1. Training and Learning. The training and learning process of the improved neural network algorithm is shown in Figure 3.

(1) The dependent variable is selected according to the actual situation, and the variables that have an impact on the dependent variable are screened out through self-organizing method so as to determine the number of input points.

(2) The number of output layer nodes is set, and the learning accuracy, the number of iteration steps, the upper limit of hidden nodes, learning parameters, and momentum coefficients are initialized.

(3) The learning sample is input to ensure that the sample value is within the specified threshold range.

(4) The values are randomly generated within the specified threshold range, and initial weight matrix is assigned.

(5) The entire network is trained according to the improved neural network algorithm. 


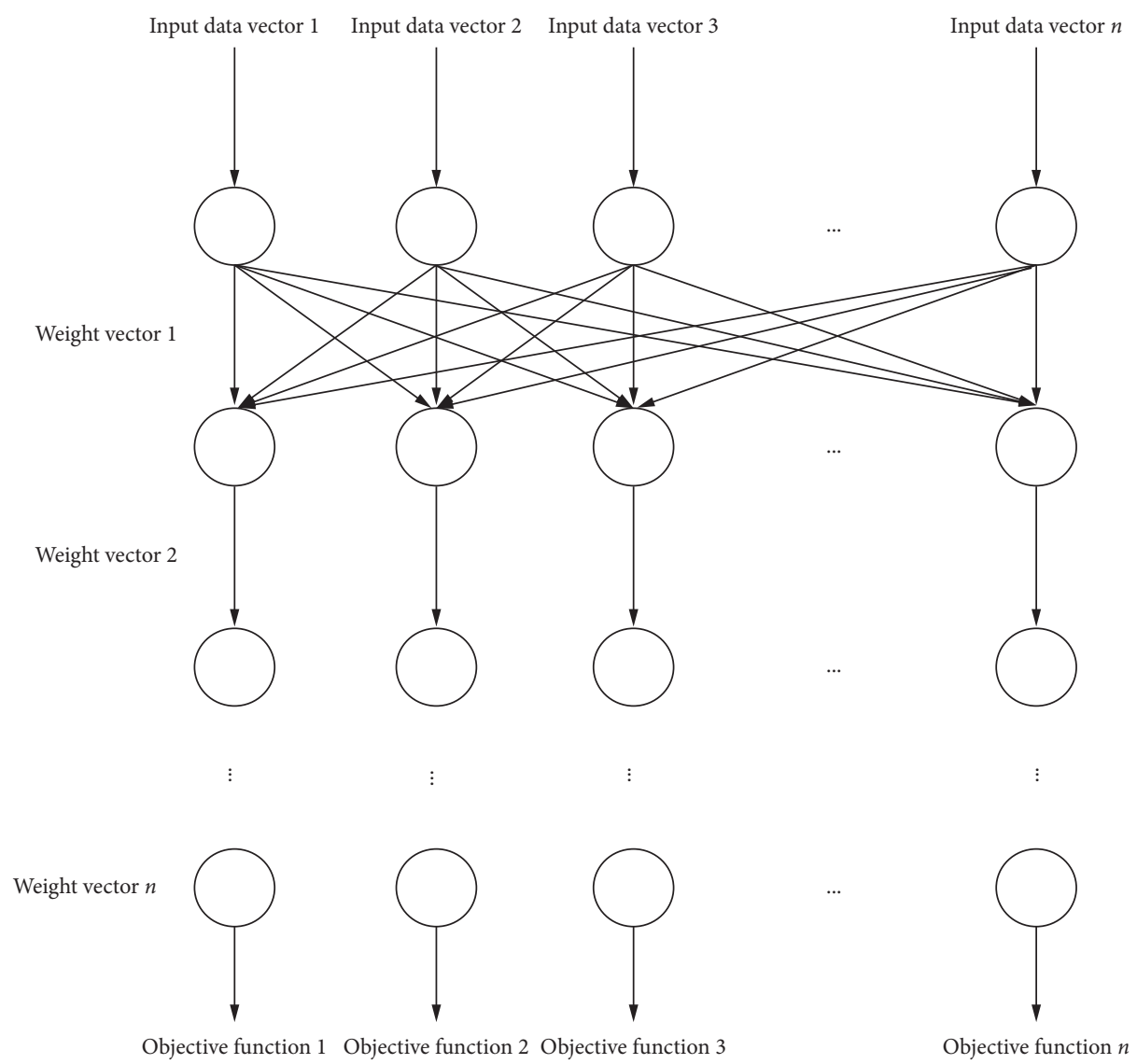

FIGURE 1: Structure of multilayer neural network.

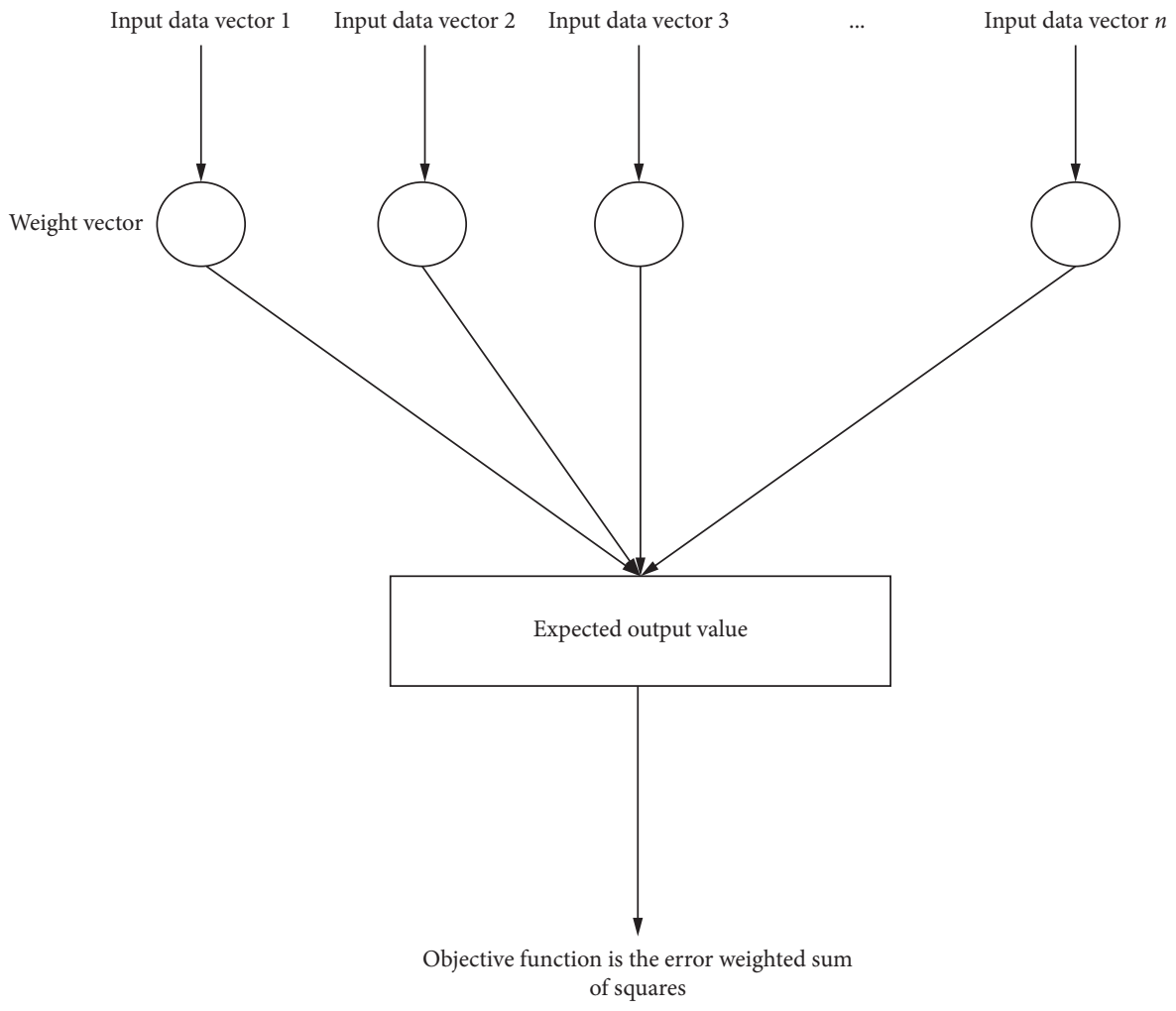

FIgURE 2: Neuron structure of neural network. 


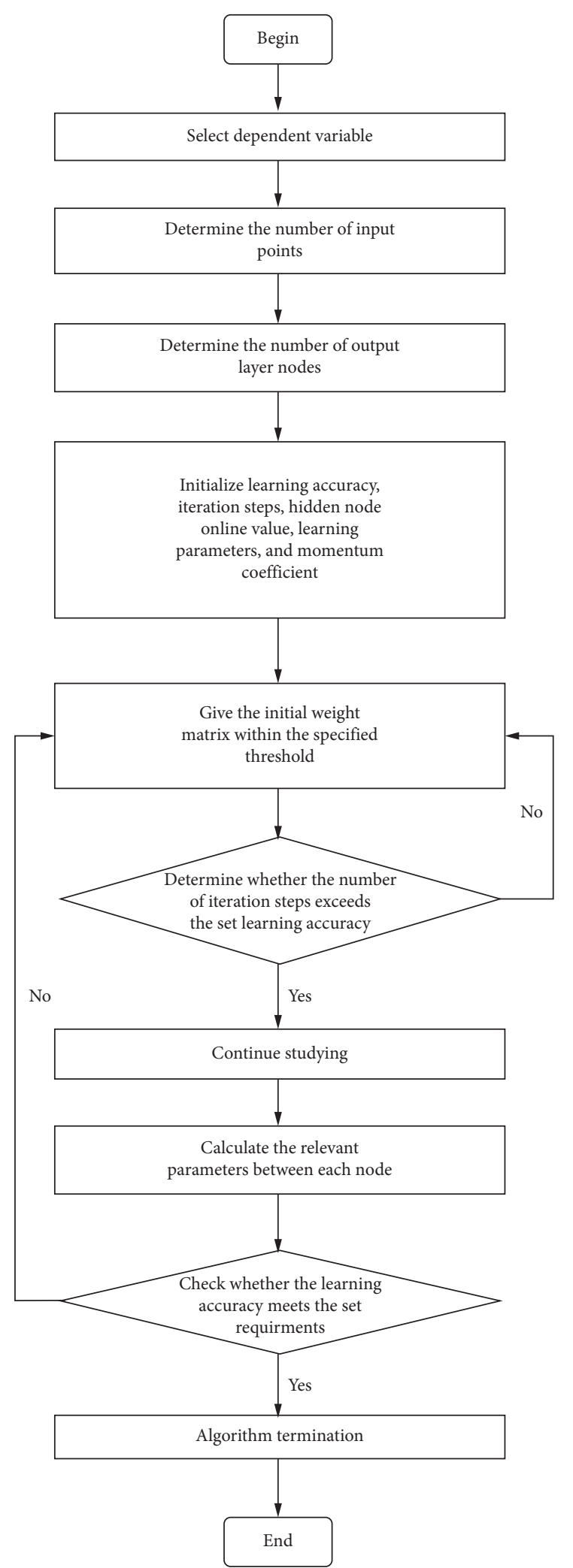

FIgURE 3: Training and learning process of improved neural network algorithm.

(6) Judge whether the number of iteration steps exceeds the set learning accuracy; if yes, continue learning; otherwise, return to the previous step.
(7) Calculate the relevant parameters between nodes and check whether the learning accuracy meets the set requirements. If yes, terminate the algorithm; otherwise, return to step (4) to complete the training and learning of the improved neural network algorithm.

4.2. Model Building. In the economic research of the fabricated building structure, the specific application steps of using the improved neural network algorithm in the mathematical analysis construction model are as follows:

(1) First, an initial dataset containing around 10-20 data is constructed. The length of the individual is the sum of the weight and the bias value of the improved neural network algorithm. The sum is multiplied by the number of digits of the improved neural network algorithm code so that the gene of a single data can be randomly selected within a predefined range.

(2) A fitness function is set to measure the authenticity of dataset. Accordingly, the fitness function is defined as a combination of different weights to generate a mean square error between the generated network output and the actual output of the corresponding sample; the two errors are superimposed to obtain the sum, and then, the economic mathematical analysis model is constructed as follows:

$$
F=\frac{\max G-\sum_{i}\left(Y_{i}-X_{i}\right)^{2}}{\max O} .
$$

In formula (4), $i$ represents the number of samples, $\max G$ represents the maximum fitness value of the neural network, max $O$ represents the maximum error that the neural network may output, $Y_{i}$ represents the sample output value after neural network learning, and $X_{i}$ represents the actual output value. The closer the neural network fitness value is to the maximum value, the higher the value of $\max G$, indicating that the dataset is real.

(3) The original dataset is selected to generate a new dataset, and the new dataset is added to another dataset.

(4) A single dataset is brought into the improved neural network algorithm after gene encoding so as to calculate the fitness of a single individual. If certain individual meets the set conditions, the application of the improved neural network algorithm ends.

(5) Arrange according to the utility degree of a single dataset to remove redundant datasets while retaining the needed datasets.

(6) Return to (3) and repeat continuously until all needed data are obtained.

The economic dataset of the fabricated building structure is determined based on all the acquired data so that the mathematical analysis is completed. 


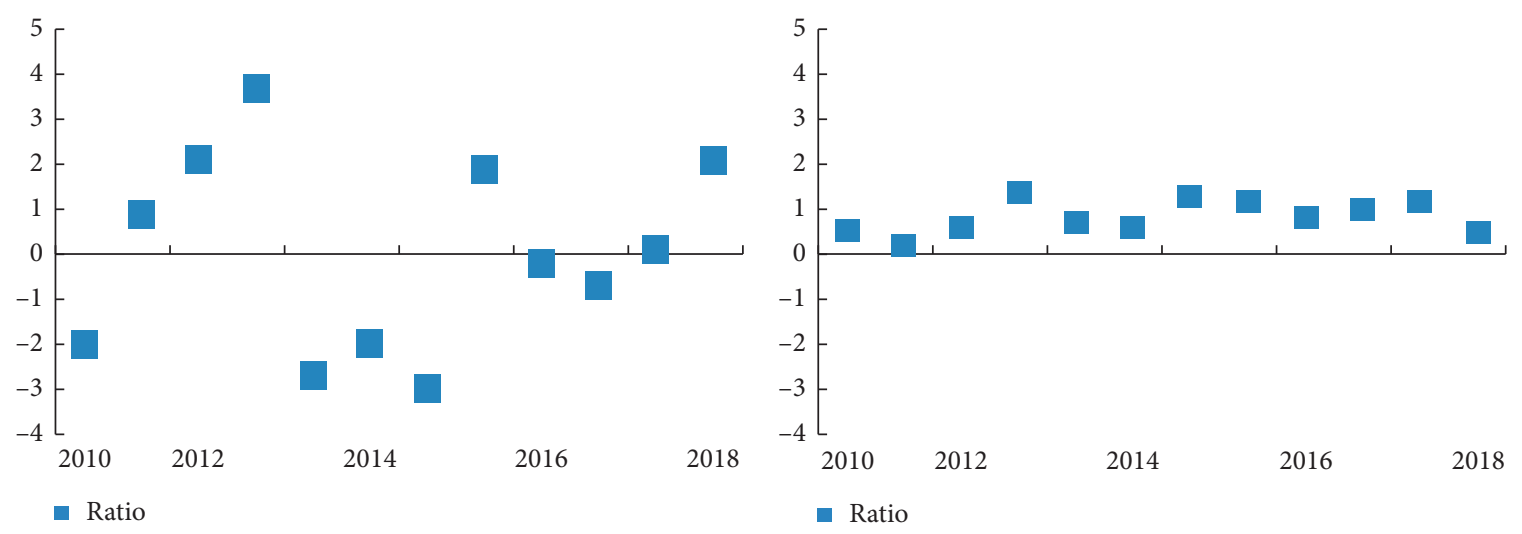

(a)

(b)

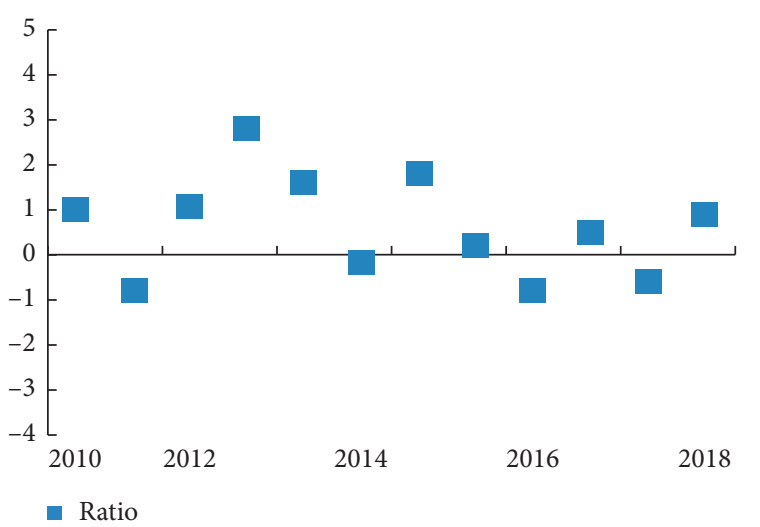

(c)

Figure 4: Relationship between added value of construction industry and other variables: (a) ratio to relative growth rate of construction area; (b) ratio to relative growth rate of total output of building industry; (c) ratio to relative growth rate of practitioners.

TABLE 1: Comparison results of analysis accuracy between two methods.

\begin{tabular}{lccc}
\hline Time/year & Gross output/million yuan & Algorithm based on neural network (\%) & Algorithm based on improved neural network (\%) \\
\hline 2010 & 105744.1 & 38 & 96 \\
2012 & 151395.1 & 43 & 95 \\
2014 & 552896.3 & 30 & 96 \\
2016 & 1238761.5 & 41 & 96 \\
2018 & 2675431.6 & 39 & 95 \\
\hline
\end{tabular}

\section{Experimental Analysis}

The actual experiments are performed so as to verify the rationality of studying the economic mathematical analysis and construction model of prefabricated building structure based on the improved neural network algorithm.

5.1. Data Analysis. Based on the prefabricated building structure in a city, the relationship between the added value of the construction industry and other variables from 2010 to 2018 is analyzed.

Figure 4(a) shows that, during 2010-2018, the added value of the construction industry has a greater impact on the construction area, and the construction area is also increasing steadily at this time; Figure 4(b) indicates that, during 2010-2018, the relative growth rate between the added value of construction industry and the total output value is stable; as shown in Figure 4(c), during 2010-2018, the relative growth rate between construction industry's added value and the number of employees is not stable.

5.2. Experimental Results and Analysis. As can be learned from the above content, the added value of the city's construction industry mainly depends on the growth of total output value but is less related to the construction area and the number of employees. On account of this phenomenon, the traditional neural network algorithm and the analysis method based on the improved neural network algorithm are used, respectively, for comparative analysis. The results are as follows.

According to Table 1, the analysis accuracy of traditional neural network algorithm is always lower than 45\%, while 
the analysis accuracy of improved neural network algorithm is always higher than $95 \%$. It can be seen that it is reasonable to research economic mathematical analysis and construction model of prefabricated building structure based on improved neural network algorithm.

\section{Conclusions}

With the rapid economic development, the stability and economics of traditional concrete building structures cannot meet the needs of modernization. Therefore, the research is based on an improved neural network algorithm for mathematical analysis and construction of fabricated building structures. The improved neural network algorithm can obtain a satisfactory economic analysis structure and provides a new effective way for future economic forecasting because it has strong generalization ability.

Despite the relatively accurate economic analysis results of this model, there are still some problems, such as less compressive analysis result due to the failure in considering the seismic performance of building structure. Therefore, the future research in this field shall focus on this aspect so as to widen the application prospects.

\section{Data Availability}

Simulation data and our model and related parameters used are provided within the article.

\section{Conflicts of Interest}

The author declares that there are no conflicts of interest.

\section{Acknowledgments}

This work was supported by the Chongqing Municipal Education Commission Science and Technology Research Project (KJQN201904003) and Research Project of Chongqing Technology and Business Institute (ZD2016-04).

\section{References}

[1] G.-H. Kim, J.-E. Yoon, S.-H. An, H.-H. Cho, and K.-I. Kang, "Neural network model incorporating a genetic algorithm in estimating construction costs," Building and Environment, vol. 39, no. 11, pp. 1333-1340, 2004.

[2] H. M. Günaydın and S. Z. Doğan, "A neural network approach for early cost estimation of structural systems of buildings," International Journal of Project Management, vol. 22, no. 7, pp. 595-602, 2004.

[3] O. Tatari and M. Kucukvar, "Cost premium prediction of certified green buildings: a neural network approach," Building and Environment, vol. 46, no. 5, pp. 1081-1086, 2011.

[4] M.-Y. Cheng, H.-C. Tsai, and E. Sudjono, "Conceptual cost estimates using evolutionary fuzzy hybrid neural network for projects in construction industry," Expert Systems with Applications, vol. 37, no. 6, pp. 4224-4231, 2010.

[5] M. W. Emsley, D. J. Lowe, A. R. Duff, A. Harding, and A. Hickson, "Data modelling and the application of a neural network approach to the prediction of total construction costs," Construction Management and Economics, vol. 20, no. 6, pp. 465-472, 2002.
[6] G.-H. Kim, J.-M. Shin, S. Kim, and Y. Shin, "Comparison of school building construction costs estimation methods using regression analysis, neural network, and support vector machine," Journal of Building Construction and Planning Research, vol. 1, no. 1, pp. 1-7, 2013.

[7] G. H. Kim, D. S. Seo, and K. I. Kang, "Hybrid models of neural networks and genetic algorithms for predicting preliminary cost estimates," Journal of Computing in Civil Engineering, vol. 19, no. 2, pp. 208-211, 2005.

[8] M. Juszczyk, "The use of artificial neural networks for residential buildings conceptual cost estimation," AIP Conference Proceedings, vol. 1558, no. 1, pp. 1302-1306, 2013.

[9] B. B. Ekici and U. T. Aksoy, "Prediction of building energy consumption by using artificial neural networks," Advances in Engineering Software, vol. 40, no. 5, pp. 356-362, 2009.

[10] M. Juszczyk, "Application of committees of neural networks for conceptual cost estimation of residential buildings," AIP Conference Proceedings, vol. 1648, no. 1, Article ID 600008, 2015.

[11] C. M. Tam and C. F. Fang, "Comparative cost analysis of using high-performance concrete in tall building construction by artificial neural networks," Structural Journal, vol. 96, no. 6, pp. 927-936, 1999.

[12] G.-H. Kim, S.-H. An, and K.-I. Kang, "Comparison of construction cost estimating models based on regression analysis, neural networks, and case-based reasoning," Building and Environment, vol. 39, no. 10, pp. 1235-1242, 2004.

[13] W. J. Zhu, W. F. Feng, and Y. G. Zhou, "The application of genetic fuzzy neural network in project cost estimate," in Proceedings of the 2010 International Conference on E-Product E-Service and E-Entertainment, pp. 1-4, IEEE, Henan, China, November 2010.

[14] M. Juszczyk and A. Leśniak, "Modelling construction site cost index based on neural network ensembles," Symmetry, vol. 11, no. 3, p. $411,2019$.

[15] H.-G. Cho, K.-G. Kim, J.-Y. Kim, and G.-H. Kim, “A comparison of construction cost estimation using multiple regression analysis and neural network in elementary school project," Journal of the Korea Institute of Building Construction, vol. 13, no. 1, pp. 66-74, 2013.

[16] V. Chandanshive and A. R. Kambekar, "Estimation of building construction cost using artificial neural networks," Journal of Soft Computing in Civil Engineering, vol. 3, no. 1, pp. 91-107, 2019.

[17] N. I. El-Sawalhi and O. Shehatto, "A neural network model for building construction projects cost estimating," A Neural Network Model for Building Construction Projects Cost Estimating, vol. 4, no. 4, 2014.

[18] U. Gulcicek, O. Ozkan, M. Gunduz, and I. H. Demir, "Cost assessment of construction projects through neural networks," Canadian Journal of Civil Engineering, vol. 40, no. 6, pp. 574-579, 2013.

[19] V. R. Ambrule and A. N. Bhirud, "Use of artificial neural network for pre design cost estimation of building projects," International Journal on Recent and Innovation Trends in Computing and Communication, vol. 5, no. 2, pp. 173-176, 2017. 